01

\title{
Динамические поляризуемости двухатомных молекул: сравнение методов ab initio и теории функционала плотности с методом замещенной функции Грина теории квантового дефекта
}

\author{
(C) А.С. Корнев, К.И. Суворов, В.Е. Чернов, И.В. Копытин, Б.А. Зон
}

Воронежский государственный университет, 394006 Воронеж, Россия

e-mail: a-kornev@yandex.ru

Поступила в редакцию 24.04.2019 г.

В окончательной редакции 19.06.2019 г.

Принята к публикации 25.06.2019 г.

Теория квантового дефекта используется для тестирования точности ab initio методов и теории функционала плотности (ТФП) при расчетах динамических поляризуемостей двухатомных молекул. Мы ограничиваемся тестированием лишь тех вариантов указанных методов, которые являются наиболее точными для расчетов статических поляризуемостей. Результаты тестирования показывают, что одна из основных погрешностей методов $a b$ initio и ТФП связана с неточностями в определении энергий возбужденных состояний, где динамические поляризуемости обладают резонансными максимумами.

Ключевые слова: динамическая поляризуемость, двухатомные молекулы, метод связанных кластеров, теория функционала плотности, теория квантового дефекта.

DOI: $10.21883 /$ OS.2019.11.48507.154-19

\section{Введение}

Поляризуемости атомов и молекул определяют их важнейшие электромагнитные свойства, такие как реакция на внешнее электромагнитное поле, ван-дерваальсовы силы, поляризационное тормозное излучение и др. Поэтому расчетам поляризуемостей посвящено огромное число публикаций (см., например, монографии и обзоры [1-5]). Отметим также недавние публикации [6-8], в которых исследовалось влияние динамических поляризуемостей на рассеяние электронов атомами, а также на туннельную ионизацию многоатомных молекул в лазерном поле.

В подавляющем большинстве теоретических работ рассчитываются статические поляризуемости, которые описывают реакцию атомов и молекул на постоянное внешнее поле. Методы расчета динамических поляризуемостей (ДП) разработаны значительно меньше. В качестве примера можно указать работы [9-13]. Здесь вполне уместно напомнить известное мнение, что показатель преломления, который определяется ДП, относится к тем величинам, которые гораздо проще определить экспериментально, чем вычислить теоретически.

Первый расчет ДП для атома водорода был выполнен аналитически с помощью кулоновской функции Грина в работах [14-16]. Расчеты ДП в основных и возбужденных состояниях атомов щелочных металлов проведены в работе [17] с помощью функции Грина в методе квантового дефекта (МКД). Мультипольные ДП и постоянные Ван-дер-Ваальса для атомов благородных газов рассчитывались в работе [18] с учетом многочастичных эффектов в приближении случайных фаз.
Метод замещенной функции Грина (МЗФГ) в МКД для расчета ДП был предложен в работе $[19]^{1}$. В этой работе было показано, что в существовавшей до этого теории МКД, в разработке которой принимали участие известные специалисты в области теоретической атомной физики [20-24], была допущена математическая ошибка. Исправление этой ошибки позволило применять МКД ко всем атомам, а не только к атомам с одним электроном сверх заполненных оболочек, таким как атомы щелочных металлов.

Применению МЗФГ к расчету ДП основных и возбужденных состояний атомов и молекул посвящены работы [25-30]. Как известно [31], МКД имеет наибольшую точность для высоковозбужденных одночастичных состояний атомов и молекул, а также для состояний непрерывного спектра вблизи первого потенциала ионизации. Хорошо известно также, что именно для таких состояний точность существующих $a b$ initio методов относительно невелика. Поэтому оба этих подхода можно считать взаимно дополняющими друг друга.

Для пояснения сути МЗФГ рассмотрим спектральное разложение функции Грина в МКД:

$$
\begin{aligned}
G_{\mathrm{QD}}\left(E ; \mathbf{r}, \mathbf{r}^{\prime}\right)= & \sum_{n} \frac{\Psi_{\mathrm{QD}}^{(n) *}\left(\mathbf{r}^{\prime}\right) \Psi_{\mathrm{QD}}^{(n)}(\mathbf{r})}{E-E_{n}} \\
& +\int d E_{c} \frac{\Psi_{\mathrm{QD}}^{(c) *}\left(\mathbf{r}^{\prime}\right) \Psi_{\mathrm{QD}}^{(c)}(\mathbf{r})}{E-E_{c}} .
\end{aligned}
$$

${ }^{1}$ В работе [19] допущена ошибка в терминологии: точка сгущения кулоновских полюсов названа там существенно особой точкой. 
Здесь $\Psi_{\mathrm{QD}}^{(n, c)}$ - волновые функции атома или молекулы в МКД в связанном $(n)$ состоянии или в континууме $(c)$, $\mathbf{r}, \mathbf{r}^{\prime}$ - координаты оптического электрона, который и определяет в основном поляризуемость. Если во внешней оболочке атома или молекулы имеется несколько эквивалентных электронов, то их вклад в поляризуемость считается равным. Аналитическое выражение для $G_{\mathrm{QD}}$ получено в работе [19] и представляет собой комбинацию функций Уиттекера, возникающих в квантовой механике при изучении движения заряженной частицы в кулоновском поле.

Для учета многочастичных эффектов, которые наиболее важны в основном и нескольких низколежащих возбужденных состояниях, вместо функции (1) строится „замещенная“ функция Грина

$$
\begin{aligned}
\mathscr{G}\left(E ; \mathbf{r}, \mathbf{r}^{\prime}\right)= & G_{\mathrm{QD}}\left(E ; \mathbf{r}, \mathbf{r}^{\prime}\right)-\sum_{n}^{N-1} \frac{\Psi_{\mathrm{QD}}^{(n) *}\left(\mathbf{r}^{\prime}\right) \Psi_{\mathrm{QD}}^{(n)}(\mathbf{r})}{E-E_{n}} \\
& +\sum_{n}^{N-1} \frac{\Psi_{\mathrm{QC}}^{(n) *}\left(\mathbf{r}^{\prime}\right) \Psi_{\mathrm{QC}}^{(n)}(\mathbf{r})}{E-E_{n}}
\end{aligned}
$$

где $\Psi_{\mathrm{QC}}^{(n)}$ - волновые функции нижних $N$ состояний, включая основное $(n=0)$, найденные методами квантовой химии (QC). Иными словами, $N$ низколежащих состояний атома или молекулы, для которых важны многочастичные эффекты, рассчитываются одним из методов QC. А далее производится замена: эти состояния, рассчитанные в МКД, заменяются состояниями, рассчитанными методами QС. При вычислениях, проведенных в работах [19,25-30], число замещенных таким образом состояний во многих атомах и двухатомных молекулах не превышало 4.

Поскольку в теории МКД и в основанном на этой теории МЗФГ точно учитываются энергии возбужденных состояний атомов и молекул, можно полагать, что в ряде случаев МЗФГ будет иметь бо́льшую точность, чем ab initio и методы теории функционала плотности (ТФП). Поэтому вполне естественно использовать метод МЗФГ для тестирования методов $a b$ initio и ТФП. Подобное тестирование для двухатомных молекул и является целью данной работы.

Как уже говорилось, в большинстве работ рассчитываются статические поляризуемости, поэтому мы также начнем сравнительный анализ методов с этого случая. В следующем разделе анализируются результаты расчета статических поляризуемостей некоторых двухатомных молекул многоконфигурационными методами, а также в рамках ТФП с различными функционалами плотности. В этом разделе не используется МЗФГ, и мы приводим соответствующие результаты отчасти для полноты. Кроме того, приводимые данные позволяют выбрать оптимальные для расчетов статической поляризуемости методы $a b$ initio и ТФП, для которых целесообразно проводить тестирование ДП. В разд. 3 сравниваются результаты расчета ДП некоторых двухатомных молекул методами ab initio и ТФП с МЗФГ. В разд. 4 приводятся основные результаты проведенного исследования.

\section{1. $A b$ initio и ТФП-расчеты статических поляризуемостей двухатомных молекул}

В настоящее время для моделирования электронной структуры молекул широко используются алгоритмы, основанные на методе гауссовых орбиталей. Они реализованы в таких вычислительных пакетах, как GAUSSIAN, DALTON, GAMESS, MOLPRO, NWCHEM, CROUP, TURBOMOLE, ORCA, PRIRODA и др.

$A b$ initio и ТФП-методы расчета статических поляризуемостей основаны на вычислении энергии молекулы как функции напряженности постоянного внешнего электрического поля и последующем дифференцировании этой энергии по напряженности поля. В пакете GAUSSIAN такое дифференцирование может выполняться как численно (например, в многоконфигурационных расчетах), так и аналитически (Хартри-Фок и многие ТФП-методы).

Анализ электронной плотности в молекулах, вычисленной с использованием 128 из известных сегодня функционалов плотности, выполнен в недавней работе [32]. Как правило, при разработке новых функционалов основной целью для теоретиков было наиболее точное воспроизведение энергий тестовых систем. Для оценки точности функционалов плотности авторы работы [32] вычисляли электронную плотность в молекулах и ее градиенты с использованием базисного набора correlation-consistent aug-cc-p $\omega \mathrm{CV} 5 \mathrm{Z}$, который несколько отличается от стандартного набора, включенного в GAUSSIAN.

Из анализа нормализованных ошибок, проведенного в работе [32], можно видеть, что вплоть до начала 2000-х гг. точность электронной плотности, получаемой новыми методами, повышалась, что отражает развитие теории от local-density approximation (LDA) к generalized gradient approximation (GGA), далее к meta-GGA и, наконец, к hybride GGA. Однако во второй декаде 2000-х гг. многие новые функционалы начинают описывать электронную плотность хуже, чем методы, разработанные в 1974 г. Основной причиной ухудшения среднего качества электронной плотности, даваемой современными функционалами, оказались так называемые сильно параметризованные методы, разработанные путем использования гибкого выражения для функционала и подгонки всех его параметров с целью лучшего воспроизведения энергий и геометрий систем в обучающем наборе. Подходы, при разработке которых использовались строгие теоретические принципы (так называемый constrained satisfaction), такой проблемы не демонстрировали. Как 


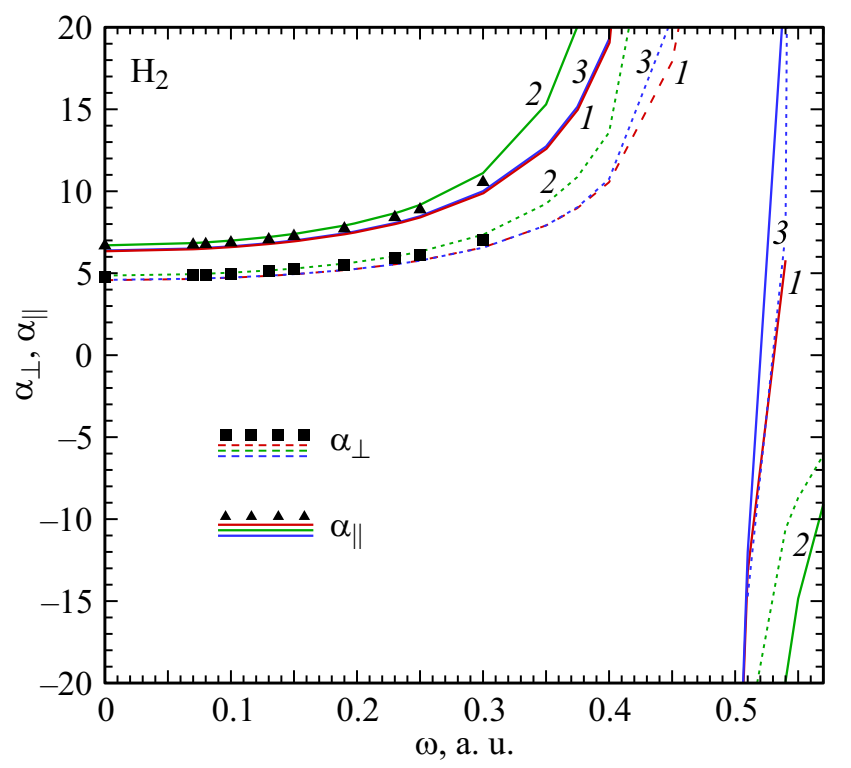

Pис. 1. Динамические поляризуемости молекулы $\mathrm{H}_{2}, \alpha_{\|}$ (сплошные кривые) и $\alpha_{\perp}$ (штриховые кривые), в а.u. $\left(a_{\mathrm{B}}^{3}\right)$ как функции частоты излучения $\omega$. Линии $1-$ расчет $a b$ initio методом $\operatorname{CCSD}(\mathrm{T})$, линии 2 - расчет с использованием ТФП (mPW1PBE), линии 3 - расчет с использованием МЗФГ. Квадратами и треугольниками показаны результаты, вычисленные в работе [51] методом суммирования по состояниям.

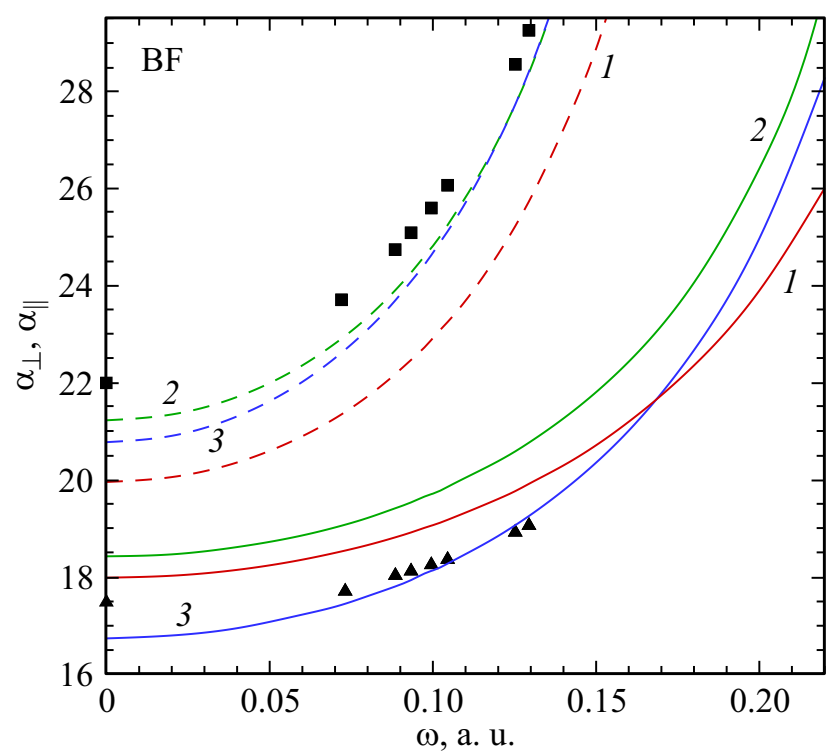

Pис. 2. То же, что на рис. 1 , но для молекулы ВF. Квадратами и треугольниками показаны результаты, вычисленные в работе [39] методом TDGI.

пишут авторы работы [32], „unconstrained functionals sacrifice physical rigor for the flexibility of empirical fitting".

Итак, согласно результатам работы [32], наилучшая точность получается при использовании одного из гибридных функционалов, предложенных Perdew и Wang [33] в 1991 г. и модифицированного Adamo и
Barone [34] в 1998 г. - mPW3PBE. Однако этот вывод не является универсальным. Многие характеристики молекул более чувствительны к выбору расчетного метода и исходной модели по сравнению с полной энергией.

В работе [35] исследованы различные многоконфигурационные методы вычисления статической электрической дипольной поляризуемости некоторых двухатомных молекул. Для расчета поляризуемостей молекул, состоящих из легких атомов неметаллов, авторы [35] рекомендуют использовать расширенные базисные наборы (Dunning's correlation-consisted X-ple-zeta (aug-cc-pVXZ), где $\mathrm{X}=\mathrm{D}, \mathrm{T}, \mathrm{Q}, 5$ или 6). Однако, несмотря на хорошее совпадение результатов, полученных в работе [35], с экспериментальными данными, многоконфигурационные методы не позволяют вычислять ДП с использованием пакета GAUSSIAN. Для этой цели пригодны лишь одноконфигурационные расчеты в приближении ХартриФока, либо стандартные методы ТФП, в рамках которых возможно получать аналитическую зависимость энергии молекулы от напряженности внешнего электрического поля.

Мы протестировали наилучшие функционалы плотности из работы [32] на расчетах статической поляризуемости легких двухатомных молекул и сравнили результаты с полученными многоконфигурационным методом $[\mathrm{RO}] \mathrm{CCSD}(\mathrm{T})$ и с имеющимися экспериментальными

Таблица 1. Статические поляризуемости некоторых двухатомных молекул (в а. u., $a_{\mathrm{B}}^{3}$ ). Для молекул $\mathrm{O}_{2}$ и $\mathrm{CN}$ использован базисный набор aug-cc-pVQZ, для остальных молекул aug-cc-pV5Z. Равновесное межъядерное расстояние $R_{e}$ взято из [38]. В первой строке для всех молекул, кроме BF, указана $\alpha_{\perp}$, во второй строке $\alpha_{\|}$. Вычисленные в работе [39] значения для BF: 1-я строка $-\alpha_{\perp}, 2$-я строка $-\alpha_{\|}, 3-я$ строка $-\bar{\alpha}$

\begin{tabular}{l|c|c|c|c|c|c|c}
\hline \multirow{2}{*}{$\begin{array}{c}\text { Моле- } \\
\text { кула }\end{array}$} & \multicolumn{2}{|c|}{$[\mathrm{RO}] \mathrm{CCSD}(\mathrm{T})$} & \multicolumn{2}{c|}{$\mathrm{mPW} 3 \mathrm{PBE}$} & \multicolumn{2}{c|}{$\mathrm{mPW} 1 \mathrm{PBE}$} & $\begin{array}{c}\text { Экспе- } \\
\text { римент }\end{array}$ \\
\cline { 2 - 8 } & $\alpha_{\perp}, \alpha_{\|}$ & $\bar{\alpha}$ & $\alpha_{\perp}, \alpha_{\|}$ & $\bar{\alpha}$ & $\alpha_{\perp}, \alpha_{\|}$ & $\bar{\alpha}$ & $\bar{\alpha}$ \\
\hline $\mathrm{H}_{2}$ & 4.57 & 5.18 & 4.89 & 5.51 & 4.86 & 5.47 & $5.42[40]$ \\
& 6.40 & & 6.75 & & 6.70 & & \\
$\mathrm{O}_{2}$ & 8.19 & 10.57 & 8.24 & 10.65 & 8.14 & 10.59 & $10.59[41]$ \\
& 15.32 & & 15.46 & & 15.50 & & \\
$\mathrm{~N}_{2}$ & 10.20 & 11.73 & 10.32 & 11.91 & 10.25 & 11.85 & $11.74[42]$ \\
& 14.79 & & 15.10 & & 15.04 & & \\
$\mathrm{CO}$ & 11.86 & 13.05 & 12.03 & 13.15 & 11.93 & 13.05 & $13.09[43]$ \\
& 15.44 & & 15.38 & & 15.28 & & \\
$\mathrm{CN}$ & 16.34 & 19.51 & 18.19 & 21.03 & 18.21 & 20.94 & $19.28[44]$ \\
& 25.85 & & 26.71 & & 26.37 & & \\
$\mathrm{HCl}$ & 16.66 & 17.22 & 17.12 & 17.62 & 16.95 & 17.47 & $17.39[45]$ \\
& 18.33 & & 18.63 & & 18.51 & & \\
$\mathrm{Cl}$ & 24.83 & 30.45 & 25.21 & 30.79 & 25.00 & 30.60 & $30.35[46]$ \\
& 41.69 & & 41.96 & & 41.79 & & \\
$\mathrm{HF}$ & 5.13 & 5.53 & 5.34 & 5.72 & 5.25 & 5.64 & \\
& 6.32 & & 6.49 & & 6.41 & & \\
$\mathrm{BF}$ & 19.95 & 19.36 & 21.29 & 20.46 & 21.16 & 20.30 & $21.99[39]$ \\
& 18.19 & & 18.81 & & 18.59 & & $17.48[39]$ \\
& & & & & & & 20.49 \\
& & & & & &
\end{tabular}


Таблица 2. Динамические поляризуемости молекулы $\mathrm{H}_{2}$ (в а. u., $a_{\mathrm{B}}^{3}$ ). ТФП-расчеты с функционалом плотности mPW1PBE выполнены в пакете GAUSSIAN, расчеты CCSD(T) - в пакете NWCHEM с использованием базисного набора aug-cс-pV5Z в обоих случаях. Равновесное межъядерное расстояние $R_{e}=0.7414 \AA$ взято из [38]. Результаты использования МЗФГ приведены из работы [29] с $N=1$ замещенным состоянием. Значения первых резонансных частот для $\alpha_{\perp}$ и $\alpha_{\|}$, равные соответственно $\omega_{\perp}=0.4858$ а. и. и $\omega_{\|}=0.4687$ а. и., взяты из работ [47-49]

\begin{tabular}{|c|c|c|c|c|c|c|c|c|}
\hline \multirow{2}{*}{$\omega$, a. u. } & \multicolumn{2}{|c|}{ ТФП } & \multicolumn{2}{|c|}{$\operatorname{CCSD}(\mathrm{T})$} & \multicolumn{2}{|c|}{ МЗФГ } & \multicolumn{2}{|c|}{$[51]$} \\
\hline & $\alpha_{\perp}$ & $\alpha_{\|}$ & $\alpha_{\perp}$ & $\alpha_{\|}$ & $\alpha_{\perp}$ & $\alpha_{\|}$ & $\alpha_{\perp}$ & $\alpha_{\|}$ \\
\hline 0.0000 & 4.86 & 6.70 & 4.57 & 6.40 & 4.59 & 6.38 & 4.74 & 6.72 \\
\hline 0.0700 & 4.94 & 6.84 & 4.65 & 6.52 & 4.66 & 6.51 & 4.82 & 6.85 \\
\hline 0.0720 & 4.95 & 6.85 & 4.65 & 6.53 & 4.67 & 6.51 & & \\
\hline 0.0800 & 4.97 & 6.88 & 4.67 & 6.56 & 4.69 & 6.54 & 4.84 & 6.89 \\
\hline 0.0834 & 4.98 & 6.90 & 4.68 & 6.57 & 4.70 & 6.56 & & \\
\hline 0.1000 & 5.04 & 6.99 & 4.73 & 6.65 & 4.74 & 6.64 & 4.90 & 7.00 \\
\hline 0.1045 & 5.05 & 7.02 & 4.74 & 6.68 & 4.76 & 6.66 & & \\
\hline 0.1300 & 5.17 & 7.21 & 4.84 & 6.84 & 4.85 & 6.83 & 5.02 & 7.20 \\
\hline 0.1363 & 5.20 & 7.26 & 4.87 & 6.89 & 4.88 & 6.88 & & \\
\hline 0.1500 & 5.29 & 7.40 & 4.94 & 7.00 & 4.95 & 7.00 & 5.12 & 7.38 \\
\hline 0.1535 & 5.31 & 7.44 & 4.96 & 7.04 & 4.96 & 7.03 & & \\
\hline 0.1900 & 5.59 & 7.91 & 5.19 & 7.44 & 5.19 & 7.43 & 5.39 & 7.86 \\
\hline 0.1979 & 5.66 & 8.03 & 5.25 & 7.54 & 5.25 & 7.54 & & \\
\hline 0.2300 & 6.03 & 8.66 & 5.54 & 8.06 & 5.54 & 8.06 & 5.78 & 8.55 \\
\hline 0.2354 & 6.10 & 8.79 & 5.60 & 8.16 & 5.60 & 8.16 & & \\
\hline 0.2500 & 6.32 & 9.17 & 5.77 & 8.47 & 5.77 & 8.47 & 6.03 & 9.01 \\
\hline 0.3000 & 7.35 & 11.1 & 6.57 & 9.94 & 6.56 & 10.0 & 6.90 & 10.7 \\
\hline 0.3500 & 9.24 & 15.3 & 7.91 & 12.7 & 7.92 & 12.7 & & \\
\hline 0.3748 & 10.9 & 20.0 & 8.97 & 15.0 & 9.02 & 15.1 & & \\
\hline 0.4000 & 13.6 & 33.4 & 10.6 & 19.1 & 10.7 & 19.3 & & \\
\hline 0.4500 & 33.8 & -8.30 & 17.9 & 58.0 & 20.6 & 58.2 & & \\
\hline 0.4800 & -308 & 17.0 & 29.8 & -74.5 & 101 & -77.4 & & \\
\hline 0.5100 & -23.1 & -207 & -203 & -13.2 & -14.7 & -12.0 & & \\
\hline 0.5400 & -10.6 & -19.9 & -22.6 & 5.79 & 7.48 & 23.4 & & \\
\hline 0.5500 & -8.70 & -14.9 & & & 130 & & & \\
\hline 0.5700 & -6.09 & -9.09 & -10.7 & -58.7 & & 25.6 & & \\
\hline
\end{tabular}

данными. В качестве референтного подхода в настоящей работе используется метод связанных кластеров $\operatorname{CCSD}(\mathrm{T})$, поскольку вклад тройных возбуждений в поляризуемость может быть существенным. Соответствующие примеры приведены в работе [36] для молекулы $\mathrm{HCl}$ и в работе [37] для $\mathrm{N}_{2}$. Результаты наших вычислений приведены в табл. 1. Здесь и далее предполагается, что ось $z$ направлена вдоль оси молекулы. Введены обозначения $\alpha_{\|} \equiv \alpha_{z z}$ и $\alpha_{\perp} \equiv \alpha_{x x}=\alpha_{y y}$ соответственно для продольной и поперечной компонент тензора поляризуемости, а также

$$
\bar{\alpha} \equiv \frac{1}{3} \operatorname{Sp} \hat{\alpha}=\frac{1}{3}\left(2 \alpha_{\perp}+\alpha_{\|}\right)
$$

для изотропной (пространственно усредненной) поляризуемости. Здесь и далее значения поляризуемостей даются в атомных единицах (а.u.), или $a_{\mathrm{B}}^{3}$, где $a_{\mathrm{B}}-$ боровский радиус. Для молекул монофторида бора (BF) экспериментальные значения поляризуемостей отсутствуют, и поэтому в табл. 1 приведены поляризуемости, вычисленные в работе [39] калибровочно-инвариантным методом решения зависящего от времени уравнения
Шредингера (,time-dependent gauge-invariant method“ TDGI).

Можно видеть весьма хорошее совпадение результатов расчета в пакете GAUSSIAN методом CCSD с экспериментальными значениями изотропной поляризуемости для большинства протестированных молекул. Незначительные отклонения наблюдаются для молекул $\mathrm{HCl}, \mathrm{Cl}_{2}, \mathrm{CN}$ и более существенные - для ВF. Возможной причиной является использование стандартных базисных наборов aug-cc-pVXZ вместо рекомендуемых в [32] aug-cc-p $\omega \mathrm{CVXZ}$.

Использование ТФП не приводит к заметному ухудшению результатов, но сокращает время расчета поляризуемости на 2-3 порядка по сравнению с многоконфигурационным методом CCSD и не требует использования суперкомпьютеров. Однако функционал $\mathrm{MPW} 1 \mathrm{PBE}$ приводит к лучшему согласию поляризуемости с экспериментом и с методом CCSD по сравнению с незначительно отличающимся от него mPW3PBE. Заметим, что в работе [32] функционал mPW3PBE признается наилучшим по среднеквадратичному отклонению электронной плот- 
Таблица 3. Динамические поляризуемости молекулы BF (в а. u., $a_{\mathrm{B}}^{3}$ ). ТФП-расчеты с функционалом плотности mPW1PBЕ выполнены в пакете GAUSSIAN, расчеты CCSD(T) - в пакете NWCHEM с использованием базисного набора aug-cс-pV5Z в обоих случаях. Равновесное межъядерное расстояние $R_{e}=1.2669 \AA$ взято из [38]. Результаты использования МЗФГ приведены из работы [30] с $N=2$ (для $\alpha_{\perp}$ ) и $N=4$ (для $\alpha_{\|}$) замещенными состояниями. Значения первых резонансных частот для $\alpha_{\perp}$ и $\alpha_{\|}$, равные соответственно $\omega_{\perp}=0.233$ а. и. и $\omega_{\|}=0.298$ а. и., взяты из справочника [50].

\begin{tabular}{|c|c|c|c|c|c|c|c|c|}
\hline \multirow{2}{*}{$\omega$, a. u. } & \multicolumn{2}{|c|}{ ТФП } & \multicolumn{2}{|c|}{$\operatorname{CCSD}(\mathrm{T})$} & \multicolumn{2}{|c|}{ МЗФГ } & \multicolumn{2}{|c|}{ [39] } \\
\hline & $\alpha_{\perp}$ & $\alpha_{\|}$ & $\alpha_{\perp}$ & $\alpha_{\|}$ & $\alpha_{\perp}$ & $\alpha_{\|}$ & $\alpha_{\perp}$ & $\alpha_{\|}$ \\
\hline 0.0000 & 21.23 & 18.43 & 19.96 & 17.99 & 20.78 & 16.74 & 21.99 & 17.48 \\
\hline 0.0200 & 21.35 & 18.47 & 20.06 & 18.03 & 20.91 & 16.80 & & \\
\hline 0.0400 & 21.71 & 18.62 & 20.36 & 18.15 & 21.31 & 16.95 & & \\
\hline 0.0600 & 22.36 & 18.86 & 20.90 & 18.36 & 22.02 & 17.23 & & \\
\hline 0.0720 & 22.91 & 19.06 & 21.35 & 18.53 & 22.62 & 17.43 & 23.71 & 17.75 \\
\hline 0.0800 & 23.35 & 19.22 & 21.72 & 18.66 & 23.10 & 17.61 & & \\
\hline 0.0885 & 23.91 & 19.42 & 22.18 & 18.82 & 23.70 & 17.81 & 24.73 & 18.03 \\
\hline 0.0933 & 24.27 & 19.54 & 22.47 & 18.92 & 24.09 & 17.94 & 25.09 & 18.12 \\
\hline 0.0995 & 24.78 & 19.71 & 22.88 & 19.06 & 24.63 & 18.13 & 25.60 & 18.25 \\
\hline 0.1000 & 24.82 & 19.72 & 22.92 & 19.07 & 24.68 & 18.14 & & \\
\hline 0.1045 & 25.25 & 19.86 & 23.25 & 19.18 & 25.12 & 18.28 & 26.07 & 18.36 \\
\hline 0.1200 & 27.00 & 20.39 & 24.65 & 19.61 & 26.98 & 18.85 & & \\
\hline 0.1252 & 27.73 & 20.59 & 25.22 & 19.77 & 27.73 & 19.07 & 28.56 & 18.92 \\
\hline 0.1294 & 28.38 & 20.77 & 25.73 & 19.92 & 28.41 & 19.26 & 29.26 & 19.06 \\
\hline 0.1400 & 30.31 & 21.27 & 27.22 & 20.30 & 30.39 & 19.79 & & \\
\hline 0.1600 & 35.74 & 22.44 & 31.25 & 21.20 & 35.78 & 21.02 & & \\
\hline 0.1800 & 45.95 & 24.07 & 38.27 & 22.36 & 45.27 & 22.68 & & \\
\hline 0.2000 & 71.49 & 26.42 & 53.19 & 23.90 & 66.05 & 24.98 & & \\
\hline 0.2200 & 246.1 & 30.15 & 105.7 & 26.01 & 148.9 & 28.29 & & \\
\hline 0.2400 & -98.58 & 37.34 & & & & 33.38 & & \\
\hline 0.2800 & -12.74 & 10.70 & & & & 60.95 & & \\
\hline
\end{tabular}

ности от значения, полученного методом CCSD. Таким образом, можно сделать вывод, что дифференциальная характеристика молекулы, примером которой является статическая поляризуемость, оказывается более чувствительной к методу расчета электронной структуры, чем значение электронной плотности.

\section{2. Расчеты ДП методами ab initio, ТФП и МЗФГ}

В настоящем разделе анализируются ДП некоторых двухатомных молекул, вычисленные с помощью МЗФГ, функционала плотности mPW1PBE в пакете GAUSSIAN и методом $\operatorname{CCSD}(\mathrm{T})$ с использованием $\Lambda$-уравнений в пакете NWCHEM.

В табл. 2 и 3 даются значения ДП соответственно для молекул $\mathrm{H}_{2}$ и $\mathrm{BF}$ в сравнении с результатами МЗФГ. Для молекулы водорода в табл. 2 также приводятся „точные“ значения поляризуемостей, вычисленные ранее в работе [51] с использованием формализма суммирования по состояниям. Эти „точные“ поляризуемости изменяются с ростом частоты $\omega$ быстрее по сравнению с рассчитанными с использованием техники МЗФГ. К сожалению, наши результаты ограничены частотами $\omega \leq 0.57$ а. и., и поэтому не могут быть сопоставлены с результатами вариационных расчетов, проведенных Адамовым и Неженцевой [52] для более высоких частот $\omega \geq 0.8$ a. u.

Для метода $\operatorname{CCSD}(\mathrm{T})$ в пакете NWCHEM наблюдается ухудшение сходимости самосогласованного поля с ростом частоты излучения $\omega$. Поэтому поляризуемости для УФ-диапазона, получаемые в пакете NWCHEM, отсутствуют в табл. 3 и 4 (см. ниже). Сходимость самосогласованного поля для методов ТФП в пакете GAUSSIAN остается удовлетворительной для всех исследованных здесь частот. Однако по мере приближения к резонансам результаты методов ТФП и ab initio начинают существенно отклоняться от результатов МЗФГ. В области резонансов ДП становятся отрицательными, что видно из табл. 2 и рис. 1 для $\mathrm{H}_{2}$. Отрицательные значения ДП в окрестности резонанса соответствуют, как известно, области отрицательной дисперсии, когда поляризуемость уменьшается при росте частоты. Следует отметить, что в окрестностях резонансов все рассмотренные здесь методы расчета ДП требуют уточнения, поскольку в этих областях необходимо учитывать колебательно-вращательную структуру уровней. Что же касается расхождения методов $a b$ initio и ТФП с результатами МЗФГ, то оно связано с погрешностями в определении энергий возбужденных состояний методами ab initio и ТФП. С практической точки зрения рас- 
Таблица 4. Динамические поляризуемости молекулы NO (в а. u., $a_{\mathrm{B}}^{3}$ ). ТФП-расчеты с функционалом плотности mPW1PBE выполнены в пакете GAUSSIAN, расчеты CCSD $(\mathrm{T})$ - в пакете NWCHEM с использованием базисного набора aug-cс-pVQZ в обоих случаях. Равновесное межъядерное расстояние $R_{e}=1.1508 \AA$ взято из [38]. Результаты использования МЗФГ приведены из работы [28] с тремя замещенными состояниями (включая основное)

\begin{tabular}{|c|c|c|c|c|c|c|c|c|}
\hline \multirow[b]{2}{*}{$\omega$, a. u. } & \multicolumn{3}{|c|}{ ТФП } & \multicolumn{3}{|c|}{$\operatorname{CCSD}(\mathrm{T})$} & \multicolumn{2}{|c|}{ МЗФГ } \\
\hline & $\begin{array}{l}\alpha_{x x} \\
\alpha_{y y}\end{array}$ & $\begin{array}{l}\alpha_{\perp} \\
\alpha_{\|}\end{array}$ & $\bar{\alpha}$ & $\begin{array}{l}\alpha_{x x} \\
\alpha_{y y}\end{array}$ & $\begin{array}{l}\alpha_{\perp} \\
\alpha_{\|}\end{array}$ & $\bar{\alpha}$ & $\begin{array}{l}\alpha_{\perp} \\
\alpha_{\|}\end{array}$ & $\bar{\alpha}$ \\
\hline 0.000 & $\begin{array}{c}9.21 \\
10.17\end{array}$ & $\begin{array}{c}9.69 \\
15.25\end{array}$ & 11.54 & $\begin{array}{l}9.20 \\
9.94\end{array}$ & $\begin{array}{c}9.57 \\
15.14\end{array}$ & 11.43 & $\begin{array}{c}9.65 \\
14.38\end{array}$ & 11.23 \\
\hline 0.015 & $\begin{array}{c}9.21 \\
10.18\end{array}$ & $\begin{array}{c}9.69 \\
15.26\end{array}$ & 11.55 & $\begin{array}{l}9.20 \\
9.95\end{array}$ & $\begin{array}{c}9.58 \\
15.15\end{array}$ & 11.44 & $\begin{array}{c}9.66 \\
14.39\end{array}$ & 11.24 \\
\hline 0.030 & $\begin{array}{c}9.22 \\
10.20\end{array}$ & $\begin{array}{c}9.71 \\
15.30\end{array}$ & 11.58 & $\begin{array}{l}9.21 \\
9.97\end{array}$ & $\begin{array}{c}9.59 \\
15.19\end{array}$ & 11.46 & $\begin{array}{c}9.69 \\
14.48\end{array}$ & 11.29 \\
\hline 0.045 & $\begin{array}{c}9.25 \\
10.25\end{array}$ & $\begin{array}{c}9.75 \\
15.37\end{array}$ & 11.62 & $\begin{array}{c}9.24 \\
10.01\end{array}$ & $\begin{array}{c}9.62 \\
15.25\end{array}$ & 11.50 & $\begin{array}{c}9.74 \\
14.64\end{array}$ & 11.38 \\
\hline 0.060 & $\begin{array}{c}9.28 \\
10.31\end{array}$ & $\begin{array}{c}9.80 \\
15.47\end{array}$ & 11.69 & $\begin{array}{c}9.27 \\
10.07\end{array}$ & $\begin{array}{c}9.67 \\
15.34\end{array}$ & 11.56 & $\begin{array}{c}9.82 \\
14.88\end{array}$ & 11.50 \\
\hline 0.0773 & $\begin{array}{c}9.34 \\
10.42\end{array}$ & $\begin{array}{c}9.88 \\
15.62\end{array}$ & 11.79 & $\begin{array}{c}9.32 \\
10.15\end{array}$ & $\begin{array}{c}9.74 \\
15.48\end{array}$ & 11.65 & $\begin{array}{c}9.94 \\
15.26\end{array}$ & 11.71 \\
\hline 0.090 & $\begin{array}{c}9.38 \\
10.51\end{array}$ & $\begin{array}{c}9.95 \\
15.76\end{array}$ & 11.89 & $\begin{array}{c}9.37 \\
10.23\end{array}$ & $\begin{array}{c}9.80 \\
15.61\end{array}$ & 11.73 & $\begin{array}{l}10.05 \\
15.62\end{array}$ & 11.91 \\
\hline 0.105 & $\begin{array}{c}9.45 \\
10.65\end{array}$ & $\begin{array}{l}10.05 \\
15.97\end{array}$ & 12.02 & $\begin{array}{c}9.43 \\
10.34\end{array}$ & $\begin{array}{c}9.89 \\
15.79\end{array}$ & 11.85 & $\begin{array}{l}10.24 \\
16.17\end{array}$ & 12.22 \\
\hline 0.120 & $\begin{array}{c}9.53 \\
10.83\end{array}$ & $\begin{array}{l}10.18 \\
16.23\end{array}$ & 12.20 & $\begin{array}{c}9.51 \\
10.48\end{array}$ & $\begin{array}{l}10.00 \\
16.01\end{array}$ & 12.00 & $\begin{array}{l}10.48 \\
16.90\end{array}$ & 12.62 \\
\hline 0.135 & $\begin{array}{c}9.62 \\
11.06\end{array}$ & $\begin{array}{l}10.34 \\
16.54\end{array}$ & 12.41 & $\begin{array}{c}9.60 \\
10.65\end{array}$ & $\begin{array}{l}10.13 \\
16.28\end{array}$ & 12.18 & $\begin{array}{l}10.86 \\
17.87\end{array}$ & 13.19 \\
\hline 0.150 & $\begin{array}{c}9.73 \\
11.35\end{array}$ & $\begin{array}{l}10.54 \\
16.93\end{array}$ & 12.67 & $\begin{array}{c}9.70 \\
10.87\end{array}$ & $\begin{array}{l}10.28 \\
16.60\end{array}$ & 12.39 & $\begin{array}{l}11.61 \\
19.18\end{array}$ & 14.13 \\
\hline 0.165 & $\begin{array}{c}9.86 \\
11.73\end{array}$ & $\begin{array}{l}10.80 \\
17.43\end{array}$ & 13.01 & $\begin{array}{c}9.82 \\
11.13\end{array}$ & $\begin{array}{l}10.48 \\
17.00\end{array}$ & 12.65 & $\begin{array}{l}17.80 \\
21.09\end{array}$ & 18.90 \\
\hline 0.1677 & $\begin{array}{c}9.88 \\
11.82\end{array}$ & $\begin{array}{l}10.85 \\
17.54\end{array}$ & 13.08 & $\begin{array}{c}9.85 \\
11.19\end{array}$ & $\begin{array}{l}10.52 \\
17.08\end{array}$ & 12.70 & $\begin{array}{l}83.46 \\
16.56\end{array}$ & 61.16 \\
\hline 0.1685 & $\begin{array}{c}9.89 \\
11.84\end{array}$ & $\begin{array}{l}10.87 \\
17.57\end{array}$ & 13.10 & $\begin{array}{c}9.86 \\
11.20\end{array}$ & $\begin{array}{l}10.53 \\
17.10\end{array}$ & 12.72 & $\begin{array}{r}-29.20 \\
16.69\end{array}$ & -13.90 \\
\hline 0.180 & $\begin{array}{l}10.01 \\
12.29\end{array}$ & $\begin{array}{l}11.15 \\
18.10\end{array}$ & 13.46 & $\begin{array}{c}9.96 \\
11.48\end{array}$ & $\begin{array}{l}10.72 \\
17.49\end{array}$ & 12.98 & $\begin{array}{c}9.33 \\
24.03\end{array}$ & 14.23 \\
\hline 0.195 & $\begin{array}{l}10.18 \\
13.19\end{array}$ & $\begin{array}{l}11.69 \\
19.05\end{array}$ & 14.14 & $\begin{array}{l}10.19 \\
11.95\end{array}$ & $\begin{array}{l}11.04 \\
18.13\end{array}$ & 13.40 & $\begin{array}{l}10.79 \\
29.99\end{array}$ & 17.19 \\
\hline 0.210 & $\begin{array}{l}10.40 \\
16.00\end{array}$ & $\begin{array}{l}13.20 \\
20.66\end{array}$ & 15.68 & $\begin{array}{l}10.32 \\
12.70\end{array}$ & $\begin{array}{l}11.52 \\
19.00\end{array}$ & 14.01 & $\begin{array}{r}11.82 \\
-80.92\end{array}$ & -19.10 \\
\hline
\end{tabular}

хождение методов расчета ДП в резонансных областях не имеет существенного значения, поскольку в этих областях можно считать, что ДП определяется единственным (резонансным) уровнем. Сравнение различных методов вычислений ДП для молекулы ВF приведено также на рис. 2.

Молекула NO (табл. 4) заслуживает отдельного рассмотрения. В ней имеет место вырождение между последней занятой (highest occupied molecular orbital, HОМО) и первой свободной (lowest unoccupied molecular orbital, LUMO) орбиталями. Данный факт протоколируется при работе пакетов NWCHEM и GAUSSIAN. Однако в протоколах не конкретизируется тип вырожденных орбиталей. Возможно, причи- ной такого вырождения является частичное заполнение $3 p \pi$-орбитали (см. [53], с. 160). Указанное вырождение HOMO-LUMO можно объяснить следующим образом. B QC-пакетах все орбитали с ненулевой проекцией орбитального момента рассматриваются как вырожденные по ее знаку. Например, $\boldsymbol{\pi}$-орбиталь рассматривается как две разные орбитали с $m= \pm 1$, каждая из которых заселяется максимум двумя электронами (с учетом спина). В молекуле NO орбиталь $3 p \pi$ заполнена лишь одним электроном (при максимально допустимой заселенности 4). Пусть это электрон с $m=+1$. Тогда $3 p \pi$-орбиталь с $m=+1$ будет играть роль НОМО, а орбиталь с $m=-1$ будет LUMO. И наоборот, в случае единственного неспаренного электрона с $m=-1$ 
роль НОМО будет играть $3 p \pi$-орбиталь с $m=-1$, а в качестве LUMO будет $3 p \pi$ орбиталь с $m=+1$.

При включении электрического поля перпендикулярно оси молекулы вырожденный уровень энергии, связанный с внешней $3 p \pi$-орбиталью, расщепляется на два подуровня, которые характеризуются разными поляризуемостями. Тем самым вырождение снимается, но при этом нарушается осевая симметрия (роль правильных функций нулевого приближения играют не состояния с $m=1$ и $m=-1$, а их комбинации, „вытянутые“ вдоль осей $x$ или $y$ ). Следовательно, равенство $\alpha_{x x}=\alpha_{y y}$ больше не имеет места. Таким образом, для частично заполненной $3 p \pi$-орбитали вычисления дают одну продольную и две различающиеся перпендикулярные компоненты поляризуемости, несмотря на осевую симметрию молекулы в отсутствие внешних полей.

Статическая компонента $\alpha_{x x}$ отличается от $\alpha_{y y}$ примерно на 10\% при вычислении практически любым методом и с любым базисным набором. Отметим, что замещающая функция основного состояния NO вычислялась в работе [28] с помощью пакета GAUSSIAN методом MP5/6-31G(d). Непосредственное вычисление статической поляризуемости таким методом в пакете GAUSSIAN дает значение, меньшее примерно в 2 раза по сравнению с МЗФГ и $10 \%$-е нарушение аксиальной симметрии в поперечных компонентах тензора поляризуемости, отмеченное выше. С увеличением частоты излучения $\omega$ указанное различие между $\alpha_{x x}$ и $\alpha_{y y}$ может нарастать. Поэтому для сравнения с МЗФГ мы дополнительно вычисляли величину

$$
\alpha_{\perp}=\left(\alpha_{x x}+\alpha_{y y}\right) / 2 .
$$

Другими словами, мы усредняли поперечные компоненты $\alpha_{x x}$ и $\alpha_{y y}$ в табл. 4. Обратим также внимание на отсутствие резонанса в тензоре поляризуемости молекулы $\mathrm{NO}$ на частоте $\omega \approx 0.1677$ а. и., связанного с возбуждением уровня $A^{2} \Sigma^{+}$, при использовании как ТФП, так и методов $a b$ initio. Данный факт, возможно, тоже обусловлен вырождением HOMO-LUMO, а также вышеупомянутой погрешностью QC-методов для энергий возбужденных состояний.

Для молекул, содержащих атомы металлов, требуются весьма специфические базисные наборы [54]. Поэтому такие молекулы здесь не анализируются, несмотря на имеющиеся ДП, полученные в работах $[29,30]$ методом МЗФГ.

\section{Заключение}

Проведенное сравнение $a b$ initio и ТФП-методов с МЗФГ позволяет оценить величину ошибки, возникающей при вычислениях ДП. Повторим, что мы сравнивали лишь те $a b$ initio и ТФП методы, которые, согласно литературным данным, наиболее пригодны для расчетов статических поляризуемостей. Совокупность этих методов не всегда совпадает с совокупностью методов, дающих наилучшие значения электронной плотности. Именно, вблизи резонансов расхождение сравниваемых подходов оказывается наиболее значительным.

Что касается конкретных результатов работы, то наиболее близкими к МЗФГ являются результаты расчетов ДП методом ab initio в пакете NWCHEM с базисным набором 6-311++G(3df,3pd) и ТФП-расчеты с функционалом плотности mPW1PBE в пакете GAUSSIAN c базисным набором aug-cc-pV5Z basis set.

Из физических соображений понятно, что МЗФГ более точно учитывает вклад возбужденных состояний атомов и молекул, а также состояний непрерывного спектра, находящихся вблизи соответствующего потенциала ионизации. Кроме того, МЗФГ точнее передает положения резонансов, вблизи которых ДП обладает максимумами. Важным достоинством МЗФГ является также возможность применения его к возбужденным состояниям $[25,27]$. Поляризуемости возбужденных атомов и молекул важны не только для расчетов штарковского сдвига спектральных линий, но и для вычисления сечений упругого (релеевского) рассеяния света. Этот вопрос важен, в частности, для астрофизики, а именно для задач переноса излучения в атмосферах звезд и планет [55-59].

Недостатком МЗФГ является прежде всего необходимость применения „ручного труда“ при проведении замены волновых функций основного и нескольких низколежащих возбужденных состояний, рассчитываемых в рамках теории квантового дефекта, на более точные волновые функции, учитывающие и многочастичные эффекты. Можно, однако, надеяться, что этот недостаток метода можно устранить путем автоматизации соответствующих алгоритмов.

\section{Благодарности}

Авторы благодарят Воронежский госуниверситет за предоставленный высокопроизводительный компьютерный центр параллельных вычислений для проведения расчетов.

\section{Финансирование работы}

Работа выполнена при финансовой поддержке Российского научного фонда (грант 19-12-00095 - расчеты поляризуемостей методами квантовой химии и сравнение базисных наборов и методов) и Министерства науки и высшего образования РФ (грант 3.4826.2017/8.9 расчеты поляризуемостей полуфеноменологическим методом).

\section{Конфликт интересов}

Авторы заявляют, что у них нет конфликта интересов. 


\section{Список литературы}

[1] Bonin K.D., Kresin V.V. Electric-dipole polarizabilities of atoms, molecules, and clusters. [S. 1.]: World Scientific, 1997. ISBN: 9789810224936

[2] Atoms, molecules and clusters in electric fields: Theoretical approaches to the calculation of electric polarizability, Ed. by Maroulis G. Computational, Numerical And Mathematical Methods In Sciences And Engineering. London: Imperial College Press, 2006. 692 p. ISBN: 1-86094-676-3

[3] Computational aspects of electric polarizability calculations: Atoms, molecules and clusters, Ed. by Maroulis G. Amsterdam: IOS Press, 2004. 535 p. ISBN: 1-58603-643-2

[4] Salam A. Molecular quantum electrodynamics: Long-range intermolecular interactions. Hoboken, New Jersey: Wiley, 2010. 399 p. ISBN: 978-0-470-25930-6

[5] Atomic and molecular nonlinear optics: Theory, experiment and computation: A homage to the pioneering work of stanisław kielich (1925-1993), Ed. by Maroulis G., Bancewicz T., Champagne B., Buckingham A.D. Amsterdam: IOS Press, 2011. 544 p. ISBN: 978-1-60750-741-3

[6] Kanya R.Laser-assisted electron scattering and diffraction in ultrashort intense laser fields / Kanya R., Morimoto Y., Yamanouchi K. // Progress in Ultrafast Intense Laser Science/ Ed. by Yamanouchi K., Paulus G., Mathur D. [S. 1.]: Springer International Publishing, 2013. V. X of Springer Series in Chemical Physics

[7] Morimoto Y., Kanya R., Yamanouchi K. // Phys. Rev. Lett. 2015. V. 115. P. 123201. doi 10.1103/PhysRevLett.115.123201

[8] Kornev A.S., Chernov V.E., Zon B.A. // Phys. Rev. A. 2017. V. 96. N 5. P. 053408. doi 10.1103/PhysRevA.96.053408

[9] Hamonou L., Hibbert A. // J. Phys. B: At. Mol. Opt. Phys. 2008. V. 41. № 24. P. 245004. doi 10.1088/09534075/41/24/245004

[10] Mitroy J., Safronova M.S., Clark C.W. // J. Phys. B: At. Mol. Opt. Phys. 2010. V. 43. № 20. P. 202001. doi 10.1088/09534075/43/20/202001

[11] Kar S. // Phys. Rev. A. 2012. V. 86. P. 062516. doi 10.1103/PhysRevA.86.062516

[12] Tang L.-Y., Bromley M.W. J., Yan Z.-C., Mitroy J. // Phys. Rev. A. 2013. V. 87. P. 032507. doi 10.1103/PhysRevA.87.032507

[13] Babb J.F. // Phys. Rev. A. 2015. V. 92. P. 022712. doi 10.1103/PhysRevA.92.022712

[14] Gavrila M.// Phys. Rev. 1967. V. 163. P. 147-155. doi 10.1103/PhysRev.163.147

[15] Rapoport L.P., Zon B.A. // Phys. Lett. A. 1968. V. 26. N 11. P. 564-565. doi 10.1016/0375-9601(68)90550-1

[16] Зон Б.А., Манаков Н.Л., Рапопорт Л.П. // ЖЭТФ. 1968. T. 55. C. 924-930.

[17] Давыдкин В.А., Зон Б.А., Манаков Н.Л., Рапопорт Л.П. // ЖЭТФ. 1971. Т. 60. С. 124-131.

[18] Амусья М.Я., Черепков Н.А., Шапиро С.Г. // ЖЭТФ. 1972. T. 63. C. $889-898$.

[19] Chernov V.E., Dorofeev D.L., Kretinin I.Y., Zon B.A. // Phys. Rev. A. 2005. V. 71. N 2. P. 022505. doi 10.1103/PhysRevA.71.022505

[20] Bates D.R., Damgaard A. // Philos. Trans. R. Soc. London, Ser. A. 1949. V. 242. N 842. P. 101-122. doi 10.1098/rsta.1949.0006

[21] Seaton M.J. // Mon. Not. R. Astron. Soc. 1958. V. 118. P. 504-518.
[22] Burgess A., Seaton M.J. // Mon. Not. R. Astron. Soc. 1960. V. 121. P. 471-473. doi 10.1093/mnras/121.5.471

[23] Fano U. // Phys. Rev. A. 1970. V. 2. P. 353-365. doi 10.1103/PhysRevA.2.353

[24] Fano U. // Phys. Rev. A. 1977. V. 15. P. 817-817. doi 10.1103/PhysRevA.15.817

[25] Chernov V.E., Dorofeev D.L., Kretinin I.Y., Zon B.A. // J. Phys. B: At. Mol. Opt. Phys. 2005. V. 38. N 13. P. 2289-2296. doi 10.1088/0943-4075/38/13/020

[26] Дорофеев Д.Л., Зон Б.А., Кретинин И.Ю., Чернов В.Е. // Опт. и спектр. 2005. Т. 99. V. 4. С. 562-566. doi $10.1134 / 1.2113366$

[27] Зон Б.А., Кретинин И.Ю., Чернов В.Е. // Опт. и спектр. 2006. V. 101. № 4. Р. 533-539. doi $10.1134 / \mathrm{S} 0030400 \mathrm{X} 06100018$

[28] Бутырский А.М., Зон Б.А. // ЖЭТФ. 2006. Т. 130. V. 3. C. $415-420$.

[29] Akindinova E.V., Chernov V.E., Kretinin I.Y., Zon B.A. // Phys. Rev. A. 2009. V. 79. N 3. P. 032506. doi 10.1103/PhysRevA.79.032506

[30] Akindinova E.V., Chernov V.E., Kretinin I.Y., Zon B.A. // Phys. Rev. A. 2010. V. 81. N 4. P. 042517. doi 10.1103/PhysRevA.81.042517

[31] Molecular applications of quantum defect theory, Ed. by Jungen C. New York: Taylor \& Francis, 1996. 664 p. ISBN: 9780750301626

[32] Medvedev M.G., Bushmarinov I.S., Sun J., Perdew J.P., Lyssenko K.A. // Science. 2017. V. 355. N 6320. P. 49-52. doi 10.1126/science.aah5975

[33] Perdew J.P., Chevary J.A., Vosko S.H., Jackson K.A. et al. // Phys. Rev. B. 1992. V. 46. P. 6671-6687. doi 10.1103/PhysRevB.46.6671

[34] Adamo C., Barone V. // J. Chem. Phys. 1998. V. 108. N 2. P. 664-675. doi 10.1063/1.475428

[35] Калугина Ю.Н., Черепанов В.Н. // Оптика атмосферы и океана. 2015. V. 28. № 5. P. 436-442. doi 10.1134/S1024856015050061

[36] Maroulis G.// J. Chem. Phys. 1998. V. 108. N 13. P. 5432-5448. doi 10.1063/1.475932

[37] Maroulis G. // J. Chem. Phys. 2003. V. 118. N 6. P. 2673-2687. doi 10.1063/1.1535443

[38] NIST computational chemistry comparison and benchmark database (release 18). http://cccbdb.nist.gov. 2018. URL: http://cccbdb.nist.gov (online; accessed: October 2018)

[39] Mérawa M., Bégué D., Rérat M., Pouchan C. // Chem. Phys. Lett. 1997. V. 280. N 3-4. P. 203-211. doi 10.1016/S00092614(97)01113-5

[40] Wormer P.E.S., Hettema H., Thakkar A.J. // J. Chem. Phys. 1993. V. 98. N 9. P. 7140-7144. doi 10.1063/1.464757

[41] Newell A.C., Baird R.C. // J. Appl. Phys. 1965. V. 36. N 12. P. 3751-3759. doi 10.1063/1.1713942

[42] Schmidt J.W., Moldover M.R. // Int. J. Thermophys. 2003. V. 24. N 2. P. 375-403. doi 10.1023/A:1022963720063

[43] Parker G.A., Pack R.T. // J. Chem. Phys. 1976. V. 64. N 5. P. 2010-2012. doi 10.1063/1.432467

[44] Medved. M., Urban M., Kellö V., Diercksen G.H. // J. Mol. Struct.: THEOCHEM. 2001. V. 547. N 1. P. 219-232. doi 10.1016/S0166-1280(01)00472-9

[45] Kumar A., Meath W.J. // Can. J. Chem. 1985. V. 63. N 7. P. $1616-1630$. doi $10.1139 / \mathrm{v} 85-272$

[46] Oddershede J., Svendsen E. N. // Chem. Phys. 1982. V. 64. N 3. P. 359-369. doi 10.1016/0301-0104(82)80004-9 
[47] Staszewska G., Wolniewicz L. // J. Mol. Spectrosc. 2002. V. 212. N 2. P. $208-212$. doi $10.1006 / j m s p .2002 .8546$

[48] Wolniewicz L., Staszewska G. // J. Mol. Spectrosc. 2003. V. 217. № 2. P. 181-185. doi 10.1016/S0022-2852(02)00047-4

[49] Wolniewicz L., Staszewska G. // J. Mol. Spectrosc. 2003. V. 220. N 1. P. 45-51. doi 10.1016/S0022-2852(03)00121-8

[50] Huber K., Herzberg G. Molecular spectra and molecular structure. iv. constants of diatomic molecules. 1 edition. New York: Van Nostrand, 1979. 716 p. ISBN: 9780442233945

[51] Bishop D.M., Pipin J. // Int. J. Quantum Chem. 1993. V. 45. N 4. P. 349-361. doi 10.1002/qua.560450403

[52] Адамов М.Н., Неженцева Е.В. // Опт. и спектр. 1972. Т. 33. V. 5. C. $850-852$.

[53] Радцииг А.А., Смирнов Б.М. Справочник по атомной и молекулярной физике. Москва: Атомиздат, 1980. 240 с.

[54] Hill J.G., Peterson K.A. // J. Chem. Phys. 2017. V. 147. N 24. P. 244106. doi 10.1063/1.5010587

[55] Bailey J., Kedziora-Chudczer L. // Mon. Not. R. Astron. Soc. 2012. V. 419. N 3. P. 1913-1929. doi 10.1111/j.13652966.2011.19845.x

[56] Vasquez M., Schreier F., Gimeno García S., Kitzmann D. et al. // Astron. Astrophys. 2013. V. 549. P. A26. doi 10.1051/0004-6361/201219898

[57] Vasquez M., Schreier F., Gimeno García S., Kitzmann D. et al. // Astron. Astrophys. 2013. V. 557. P. A46. doi 10.1051/0004-6361/201220566

[58] Chance K., Martin R.V. Spectroscopy and radiative transfer of planetary atmospheres. [S. 1.]: Oxford University Press, 2017. ISBN: 9780199662104

[59] Tinetti G., Encrenaz T., Coustenis A. // Astron. Astrophys. Rev. 2013. V. 21. N 1. P. 63. doi 10.1007/s00159-013-0063-6 